\title{
Analysis of the feasibility of microwave ablation for colorectal liver metastases: a preliminary report
}

\author{
Yiren $\mathrm{Hu}^{1}$, Zhangwei Yang ${ }^{2}$, Yuesheng Sun ${ }^{2}$ \\ ${ }^{1}$ Department of General Surgery, Third Affiliated Hospital of Soochow University, Changzhou, Jiangsu, China \\ ${ }^{2}$ Department of General Surgery, Wenzhou People's Hospital, Wenzhou, China
}

Videosurgery Miniinv 2020; 15 (1): 30-35

DOI: https://doi.org/10.5114/wiitm.2019.86921

\begin{abstract}
Introduction: Colorectal liver metastasis is a challenge in the treatment of colorectal cancer and an important factor affecting the prognosis of patients. Currently, microwave ablation has gradually been applied for the treatment of liver cancers as a type of thermal ablation. However, there are no large-scale studies on the effectiveness of microwave ablation for colorectal liver metastases.

Aim: To investigate the efficacy of microwave ablation and liver resection for liver metastases from colorectal cancer, and to compare the prognosis between patients treated with microwave ablation and those in the SEER (SUrveillance, Epidemiology, and End Results, National Cancer Institute) database.

Material and methods: We retrospectively analyzed the clinical data of 24 patients with colorectal liver metastasis who underwent radical colorectal cancer resection and liver microwave ablation (the MWA group) and 12 patients who received radical colorectal cancer resection and liver resection (the $L R$ group). The complete ablation rate and complications after microwave ablation were observed. Survival analysis was performed for cases treated with liver resection and cases from the SEER database.

Results: A total of 53 tumors were ablated in the 24 patients who underwent radical colorectal cancer resection and liver microwave ablation; 52 tumors achieved complete ablation after the first ablation (98.1\%). No serious complications occurred in the MWA group, and long-term survival was not significantly different between the MWA and other groups. Conclusions: Microwave ablation for colorectal liver metastases avoids extensive liver resection while ensuring therapeutic efficacy; the operation is safe, feasible, and reproducible.
\end{abstract}

Key words: liver metastases of colorectal cancer, microwave ablation, SEER data.

\section{Introduction}

Colorectal cancer is the fourth most common malignancy in the world, and liver metastases eventually appear in over $50 \%$ of colorectal cancer patients [1]. According to statistics, approximately $10-25 \%$ of colorectal cancer patients have liver metastases at the time of diagnosis, and approximately $18-37 \%$ of patients have liver metastases after radical colorectal cancer resection [2]. Colorectal liver metastasis is a challenge in the treatment of colorectal cancer and an important factor affecting the prognosis of patients. Radical colorectal cancer resection with simultaneous resection of liver lesions is feasible for resectable single or multi-focal liver metastases. Nevertheless, many patients lose the opportunity of undergoing surgery because the liver metastases cannot be removed. Currently, microwave ablation is gradually being applied for the treatment of liver cancers as a type of thermal abla-

\section{Address for correspondence}

Dr. Yuesheng Sun, Department of General Surgery, Wenzhou People's Hospital, 57 Canghou Street, Wenzhou 325000, China,

e-mail: sunyuesheng886@163.com 
tion. It has the advantages of being minimally traumatic and very safe, and ablation can be performed for lesions at sites where resection is difficult. However, there are no large-scale studies on the effectiveness of microwave ablation for colorectal liver metastases.

\section{Aim}

In this study, we aimed to determine the safety, local efficacy, and long-term prognosis of microwave ablation for colorectal liver metastases. In addition, the survival difference was compared between patients treated with microwave ablation and those treated with liver resection as well as those in the SEER database.

\section{Material and methods}

\section{General data}

Between April 2012 and April 2017, 24 patients with colorectal liver metastases underwent radical colorectal cancer resection and microwave ablation of liver metastases at our hospital (MWA group), and 12 patients underwent colorectal cancer resection and resection of liver metastases (LR group). Diagnoses in this study were based on the postoperative pathology of colorectal cancer as well as imaging and laboratory data of any discovered liver metastases. The inclusion criteria were as follows: (1) having colorectal liver metastasis resectable via surgery, (2) absence of extrahepatic metastatic lesions, (3) and providing informed consent to participate in the study. All patients underwent detailed preoperative examination and tumor morphological studies using computed tomography (CT) and/or magnetic resonance imaging (MRI) (Photo 1). Three control groups (1-SEER, 2-SEER, and 3-SEER) were identified from the SEER database through propensity score matching (nearest method) based on age, tumor, operation type, race, TNM, and other factors.

\section{Instruments and equipment}

(1) Ultrasound equipment: UltraView Pro Focus2202 (Denmark); (2) Laparoscopic ultrasound
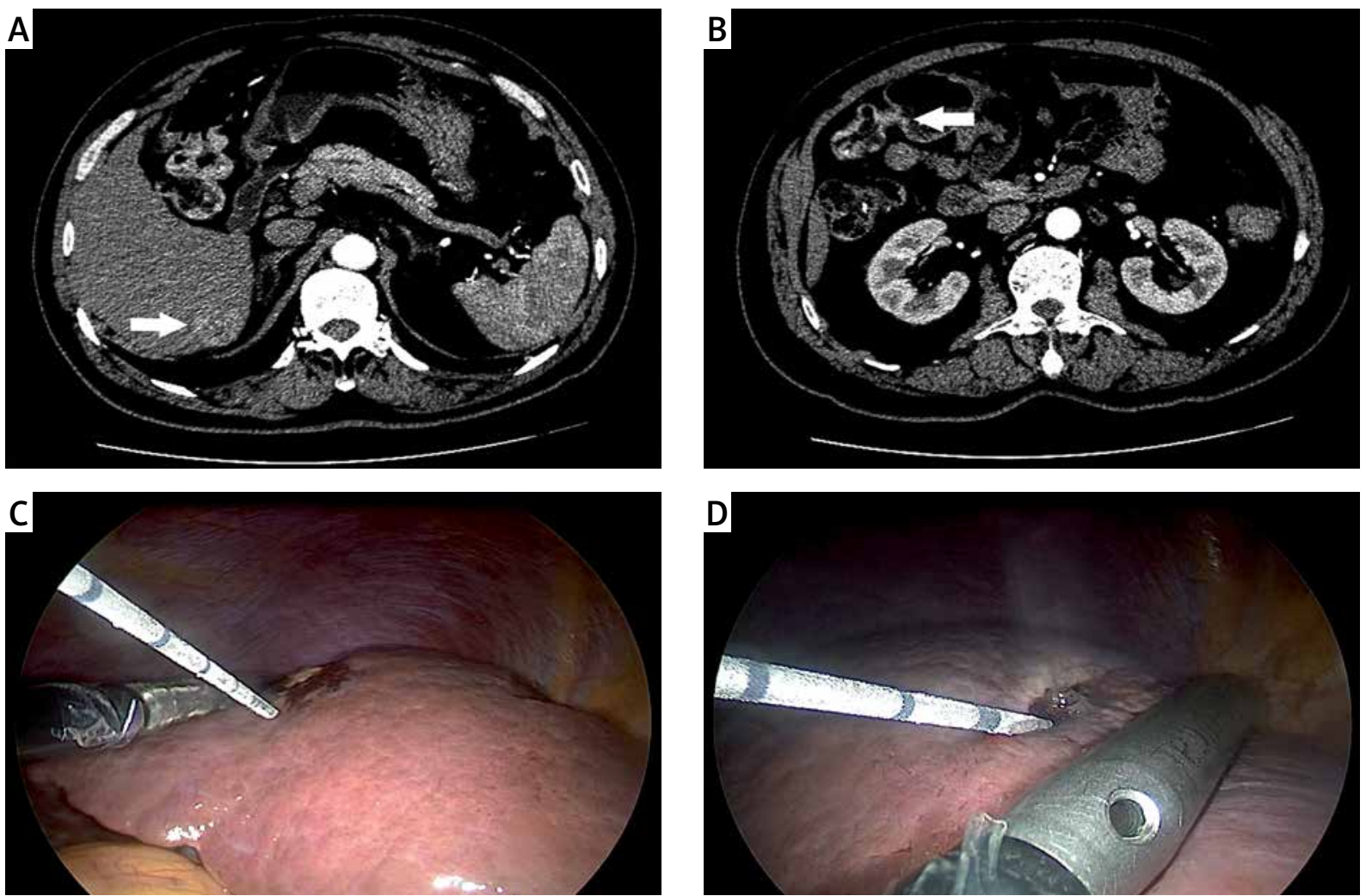

Photo 1. Preoperative contrast-enhanced CT examination: location of hepatic metastases (A); location of primary tumor (B). Intraoperative photo showing the procedure of laparoscopic MWA (C, D) 
probe: BK four-way laparoscopic probe 8666-RF (UK), end bendable soft probe; (3) Microwave therapy instrument: Nanjing Qinghai Co., Ltd. (China), MTC-3C microwave coagulation therapy device, microwave frequency $2450 \pm 50 \mathrm{~Hz}$, power 0-100 W.

\section{Ablation treatment method}

Under the guidance of the ultrasound probe, a microwave needle electrode was used to penetrate the tumor tissue. The microwave needle electrode was confirmed to be located at the center of the tumor, and the ablation power was set at 80-100 W. The ablation time was set to 4-10 min depending on the condition of the patient (Photo 1). For tumor lesions $\leq 3 \mathrm{~cm}$, a single-needle-based tumor-centered ablation method was used. For tumors $>3 \mathrm{~cm}$ but $\leq 5 \mathrm{~cm}$, a multi-needle spot ablation method was used. For tumors $>5 \mathrm{~cm}$, a multi-needle site and staged tumor reduction ablation method was used. After ablation, radical colorectal cancer treatment was performed.

\section{Postoperative follow-up and efficacy assessment}

Follow-up was conducted until July 2017. The follow-up rate was $83.3 \%(20 / 24)$. All patients were followed up via telephone and outpatient visits. The efficacy assessment criteria for ablation were as follows: all necrotic treatment areas showed lower density shadows than the original tumors and there were cystic low-density necrotic areas. Enhanced MRI/CT showed no enhancement in the tumor area.

\section{Statistical analysis}

Data analysis was performed using $R$ software. The overall survival rate was calculated and the survival curve was plotted using the Kaplan-Meier method. The R language "Matchlt" package was used to perform propensity score matching, and the nearest neighbor matching method was used for matching.

\section{Results}

\section{Patient characteristics}

Of the 24 patients treated with radical colorectal cancer treatment and liver microwave ablation (MWA group), 14 were men and 10 were women. The median age was 59 years (range: $34-79$ years). Sixteen patients underwent laparoscopic surgery and 8 were treated with open surgery. Of the 12 patients in the LR group, 6 were men and 6 were women. Two patients underwent laparoscopic surgery and 10 were treated with open surgery. We obtained data from the SEER database, and after matching by age, sex, ethnicity, tumor stage, chemotherapy, and other factors, 3 control groups with a total of 72 patients were matched to evaluate the survival. The detailed data are shown in Table I.

\section{Postoperative complications}

A total of 53 tumors were treated among the 24 patients in the MWA group; complete ablation was achieved after the first treatment in 52 patients, with a complete ablation rate of $98.1 \%$ (52 of 53 patients). In the remaining patient, percu-

Table I. Demographic data for each group

\begin{tabular}{|c|c|c|c|c|c|}
\hline Parameter & WA & LR & 1-SEER & 2-SEER & 3-SEER \\
\hline Age [years] & $58(34-79)$ & $56(37-75)$ & $59(36-79)$ & $58(34-79)$ & $58(35-79)$ \\
\hline Sex (female/male) & $10 / 14$ & $6 / 6$ & $9 / 15$ & $10 / 14$ & $9 / 15$ \\
\hline Surgery (laparoscopic/open) & $16 / 8$ & $2 / 10$ & N & N & $\mathrm{N}$ \\
\hline Primary tumor location (colon/rectum) & $20 / 4$ & $9 / 3$ & $20 / 4$ & $20 / 4$ & $20 / 4$ \\
\hline Number of liver metastases & 53 & 29 & N & $\mathrm{N}$ & $\mathrm{N}$ \\
\hline Incomplete ablation & 1 & 0 & $\mathrm{~N}$ & $\mathrm{~N}$ & $\mathrm{~N}$ \\
\hline \multicolumn{6}{|l|}{ Tumor size $[\mathrm{cm}]$ : } \\
\hline$<3$ & 39 & 20 & $\mathrm{~N}$ & $\mathrm{~N}$ & $\mathrm{~N}$ \\
\hline$\geq 3$ & 14 & 9 & & & \\
\hline Number lost to follow-up & 4 & 3 & 0 & 0 & 0 \\
\hline
\end{tabular}

$N$ means - not reported. 
taneous microwave ablation was performed after tumor surgery, and ultrasound contrast confirmed that the tumor was inactive. The technical success rate was $100 \%$. In the MWA and LR groups, there were no postoperative complications, severe adverse reactions, or perioperative deaths. Of the 24 patients in the MWA group, 1 patient developed a liver abscess and 2 developed pleural effusion, which improved after treatment. Among the 24 patients, secondary adverse reactions including fever were observed in 5 (20.8\%), abdominal distension in 3 (12.5\%), and abdominal pain in 14 (58.3\%); the complications recovered within 7 days (Table II).

\section{Survival time and survival analysis}

The median survival time of patients after microwave ablation was 12 months, and the overall 1-year and 2 -year survival rates were $66.7 \%$ and $54.2 \%$, respectively. The median survival time of the LR group was 11 months, and the 1- and 2-year survival rates were $41.7 \%$ and $25 \%$, respectively. The results of Kaplan-Meier survival analysis showed that the survival curves did not differ significantly between the MWA and LR groups $\left(\chi^{2}=2.2, p=0.1\right)$. The $\chi^{2}$ value was $0.3(p=0.6)$ when data were compared with those of the 3 SEER groups, suggesting that there was no significant difference in prognosis between the MWA and SEER database groups (Figure 1).

\section{Discussion}

Surgical resection is the preferred treatment for colorectal liver metastases, but only $20-25 \%$ of patients have the opportunity to undergo surgery [1, 2]. If colorectal liver metastases are not treated effectively, the median survival of patients with colorectal liver metastases is only 6-12 months [3]. The primary purpose of treatment of patients with colorectal liver metastases is to control the progression of intrahepatic tumors, prolong the survival time of patients, and improve the quality of life [4]. Prospective randomized controlled clinical trials and retrospective comparative studies have shown that local ablation (radiofrequency ablation or microwave ablation) can achieve long-term survival comparable to surgical resection for small hepatocellular carcinoma. However, large-scale studies on microwave ablation of colorectal liver metastases have not been performed.

The local residual tumor rate is an important factor that can be used for determining the reliability of this
Table II. Postoperative complications of MWA and LR group

\begin{tabular}{|lcc|}
\hline Variable & WA & LR \\
\hline Liver abscess & 1 & 0 \\
\hline Bile leakage & 0 & 3 \\
\hline Abdominal distension & 3 & 2 \\
\hline Abdominal pain & 14 & 10 \\
\hline Postoperative bleeding & 0 & 1 \\
\hline Ascites & 1 & 2 \\
\hline Pleural fluid & 2 & 2 \\
\hline Fever & 5 & 3 \\
\hline Other & 0 & 2 \\
\hline
\end{tabular}

treatment. Previous studies have found that the efficacy of microwave ablation therapy is determined by whether the coagulation zone during microwave $a b$ lation therapy completely covers the two-dimensional space of the entire tumor $[5,6]$. In order to reduce the residual cancer around the tumor, the coagulation necrotic area should cover $5 \mathrm{~mm}$ beyond the outer margin of the tumor [7]. Therefore, for a tumor with a diameter of $\leq 3 \mathrm{~cm}$, the microwave antenna is in the center of the tumor, and the treatment can be completed with one coagulation cycle. For a tumor with a diameter of $>3 \mathrm{~cm}$, multiple points and multi-directional punctures are required, and treatment must be divided into parts [8-10]. In this study, a total of 53 patients underwent microwave ablation, of whom 39 had tumors $<3 \mathrm{~cm}$ and 14 had tumors $>3 \mathrm{~cm}$. If local recurrence occurred in the ablation zone or a new lesion developed in the adjacent liver tissue, B-scan ultrasound-guided percutaneous microwave ablation was performed. The present study showed that there was no residual tumor for tumors $\leq 3 \mathrm{~cm}$, and that there was 1 site of a residual tumor in 1 case among 14 tumors $>3 \mathrm{~cm}$. Re-examination using enhanced CT 1 month after B-scan ultrasound-guided percutaneous microwave ablation showed no residual tumors. In addition, no obvious in situ recurrence was observed during the follow-up of the MWA group. The low residual tumor rate and lack of in situ recurrence also confirmed the efficacy of ablation in the present study.

In cancer patients, recommendations about treatment depend on the survival time. In the present study, we performed a comparative analysis between the MWA and LR groups in our hospital. The results were not significantly different between the MWA and LR groups. The effect of MWA primarily 


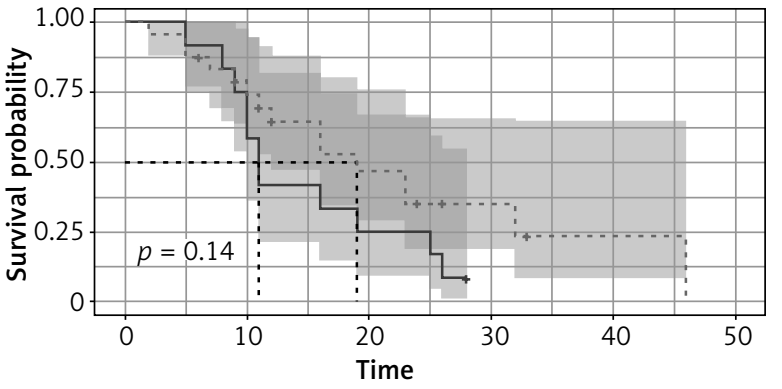

Number at risk

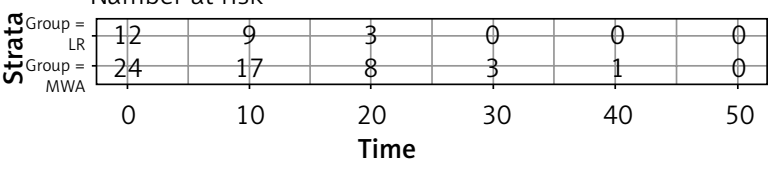

Strata + Group $=\mathrm{LR} \quad+$ Group $=$ MWA

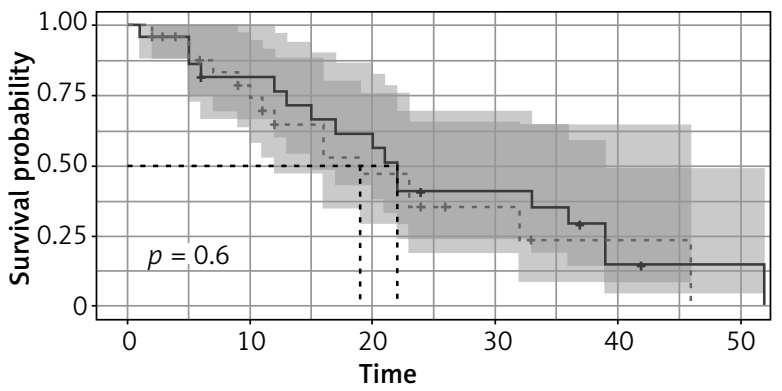

Number at risk

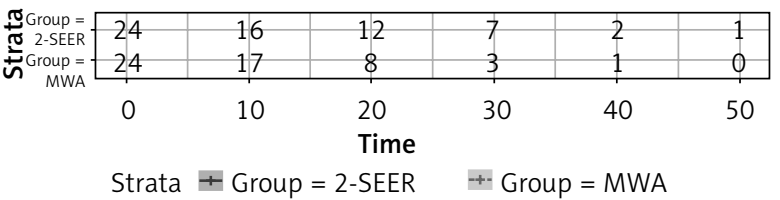

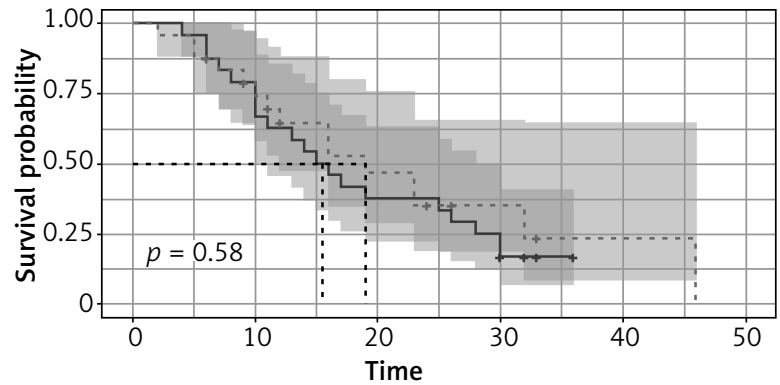

Number at risk

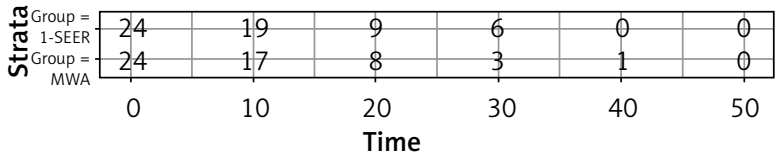

Strata + Group $=1$-SEER $\quad+$ Group $=$ MWA

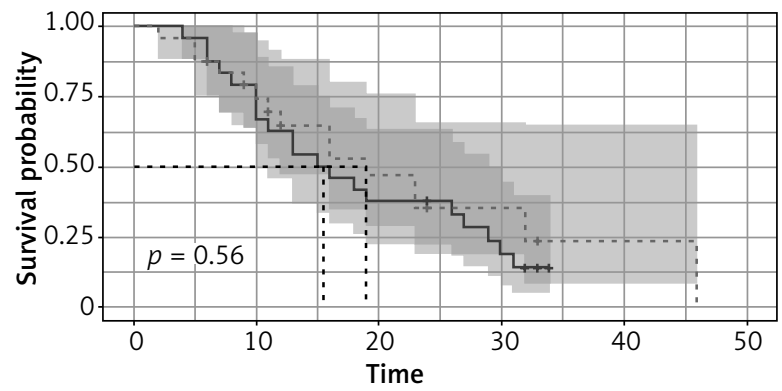

Number at risk

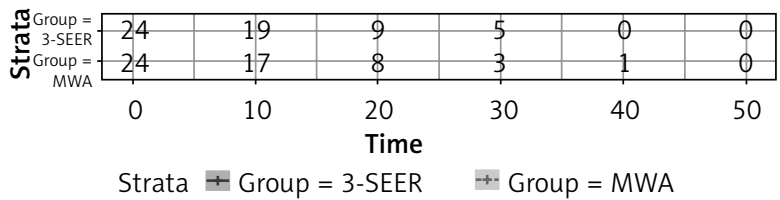

Strata + Group $=3$-SEER + Group $=$ MWA

Figure 1. Kaplan-Meier analysis for MWA group compared with control groups

depends on the exact local treatment outcomes of microwave ablation: low residual tumor rate and no recurrence in situ. Furthermore, with MWA, intrahepatic re-ablation can be performed for metastatic tumor recurrences; greater liver reserve and operating space were observed in the MWA group than in the LR group. This ability for re-operation is extremely beneficial for comprehensive postoperative treatment of patients with colorectal liver metastases, and thus, alternative surgical treatments for patients with recurrence are still available. In addition, for metastases to specific sites, such as the first hepatic hilum, second hepatic hilum, and deep in the liver, the surgical procedure is more difficult and more of the liver is resected; this results in higher risks and the possibility of more serious complications [11, 12]. Ablation eliminates these risks while completely curing metastases and preserving a large amount of the normal liver tissue [8-10]. These characteristics are responsible for less frequent postoperative complications in the MWA group; the incidence rates of bile leakage, abnormal liver function, abdominal pain, abdominal distension, and abdominal infection were significantly reduced in the MWA group. In addition, patients recovered quickly after surgery, resulting in rapid rehabilitation $[13,14]$. Owing to the small number of cases in the resection group, the present study included a large amount of data from the SEER database; the data of 1300 Asian patients with colorectal liver metastasis were extracted, and propensity score matching based on various factors yielded 3 groups. The comparison showed that survival did not differ significantly between the WMA and SEER database groups. This also demonstrates that microwave ablation of colorectal liver metastases as a local treatment method can avoid liver resection and reduce postoperative complications while achieving the same prognosis as observed in 
the SEER data. However, this study only evaluated a small number of cases and only involved preliminary empirical analysis; thus, the results of longterm prognosis analysis may have some defects.

In summary, microwave ablation as a local treatment technique for liver tumors has many advantages over liver resection, such as reproducibility, minimal trauma, and fewer adverse reactions and complications. Microwave ablation can also serve as the best choice for patients with liver metastases, which are more common in colorectal cancer, especially in patients with no indications or tolerance for surgery. For patients with resectable liver metastases, ablation can also achieve the same therapeutic effect and survival time as resection. In addition, there is a great improvement in the quality of life, and at the same time, it provides better treatment conditions for patients with recurrences. Therefore, microwave ablation can be one of the treatment options for liver lesions in patients with colorectal liver metastases. However, microwave ablation therapy is categorized as a local treatment, and colorectal liver metastases are primarily hematogenous metastases. Thus, microwave ablation along with systemic chemotherapy is necessary to kill cancer cells in the circulation, inhibit tumor recurrence, and control tumor progression.

This study has several limitations. First, the overall number of cases is small, and it is not a multi-center study. Secondly, the volume of metastases in the MWA group was smaller than that in the LR group, which is also likely to lead to a difference in prognosis. Finally, the SEER database data do not clearly label the treatment of liver metastases, and there may be untreated patients among the cases, resulting in differences in survival curves. Nevertheless, there are only a few studies on microwave ablation, and treatment efficacy has not been verified by largescale data. Therefore, the short-term and long-term results of the present study will provide new ideas and further investigation regarding the treatment of colorectal liver metastases.

\section{Conclusions}

Microwave ablation is a safe and effective treatment for colorectal liver metastases. It can effectively control intrahepatic progression of liver metastases, and it has advantages over liver resection in terms of short-term efficacy. Therefore, in clinical practice, microwave ablation of colorectal liver metastases avoids extensive liver resection and improves the quality of life of patients; it can be chosen as a minimally invasive surgery technique.

\section{Conflict of interest}

The authors declare no conflict of interest.

\section{References}

1. Steele G Jr, Ravikumar TS. Resection of hepatic metastases from colorectal cancer. Biologic perspective. Ann Surg 1989; 210: 127-38.

2. Dupre A, Gagniere J, Chen Y, et al. Management of synchronous colorectal liver metastases. Bull Cancer 2013; 100: 363-71.

3. Bengtsson G, Carlsson G, Hsfstrom L, et al. Natural history of patients with untreated liver metastases from colorectal cancer. Am J Surg 1981; 141: 586-9.

4. Lencioni R. Loco-regional treatment of hepatocellular carcinoma. Hepatology 2010; 52: 762-73.

5. Jagad RB, Koshariya M, Kawamoto J, et al. Laparoscopic microwave ablation of liver tumors: our experience. Hepatogastroenterology 2008; 55: 27-32.

6. Bhardwj N, Strickland AD, Ahmad F, et al. Microwave ablation for unresectable hepatic tumours: clinical results using a novel microwave probe and generator. Eur J Surg Oncol 2010; 36: 264-58.

7. Correa-Gallego C, Fong Y, Gonen M, et al. A retrospective comparison of microwave ablation vs. radiofrequency ablation for colorectal cancer hepatic metastases. Ann Surg Oncol 2014; 21 : 4278-83.

8. Yang B, Li Y. A comparative study of laparoscopic microwave ablation with laparoscopic radiofrequency ablation for colorectal liver metastasis. J Buon 2017; 22: 667-72.

9. Tiong L, Maddern GJ. Systematic review and meta-analysis for survival and disease recurrence after radiofrequency ablation hepatocellular carcinoma. Br J Surg 2011; 98: 1210-24.

10. Primrose J, Falk S, Finch-Jones M, et al. Systemic chemotherapy with or without cetuximab in patients with resectable colorectal liver metastasis: the New EPOC randomised controlled trial. Lancet Oncol 2014; 15: 601-11.

11. Gruenberger B, Scheithauer W, Punzengruber R, et al. Importance of response to neoadjuvant chemotherapy in potentially curable colorectal cancer liver metastases. BMC Cancer 2008; 8:120.

12. Lalmahomed ZS, Ayez N, van der Pool AE, et al. Anatomical versus nonanatomical resection of colorectal liver metastases: is there a difference in surgical and oncological outcome? World J Surg 2011; 35: 656-61.

13. Rong GH, Bai WL, Dong Z, et al. Long-term outcomes of percutaneous cryoablation for patients with hepatocellular carcinoma within Milan criteria. PLoS One 2015; 10: e0123065.

14. Xu Z, Yang Z, Pan J, Hu Y. Individualized laparoscopic B-ultrasound-guided microwave ablation for multifocal primary liver cancer. Videosurgery Miniinv 2018; 13: 9-16.

Received: 5.05.2019, accepted: 6.06.2019. 